\title{
Collecting and Analysing - 1.6eV - 20keV Emission Spectra in an EPMA
}

\author{
C.M. MacRae ${ }^{1}$, N.C. Wilson ${ }^{1}$, A. Torpy ${ }^{1}$, J. Bergmann ${ }^{2}$ and H. Takahashi ${ }^{3}$ \\ 1. CSIRO Mineral Resources, Private Bag 10, Clayton South, Vic, Australia \\ 2. Bruker Pty Ltd, PO Box 8432, Northland Centre, Vic, Australia \\ 3. JEOL Ltd., 3-1-2 Musashino, Akishima, Tokyo, Japan
}

Advances in detector technology are lowering the energy of x-rays that can be measured, opening exciting new possibilities for microanalysis [1, 2]. Energy dispersive spectrometers (EDS) can now detect X-rays with energies as low as Mg-L (49eV), while the soft x-ray emission spectrometer (SXES) can measure similarly low energies through to $350 \mathrm{eV}$ but with much higher energy resolution.

The process of soft x-ray generation involves transitions from electron orbitals that are involved in bonding, and thus the peak shape and position of soft x-ray lines are sensitive to the local chemical environment. X-ray transitions from KLM and $\mathrm{N}$ shell are now routinely observed and in the case of SXES higher order reflections are commonly observed. This creates both problems and benefits for microanalysis: quantification becomes problematic when the x-ray line shape and position varies with composition, but the x-ray line shape and position carry information about the chemical bonding allowing the microanalysis to yield more than just the chemical concentrations.

At lower photon energies, cathodoluminescence (CL) is sensitive to both material composition and structure of the host lattice, as it originates from effects such as conduction to valence transitions and phonon modes. Combining the collection of SXES, EDS, wavelength dispersive spectrometers and CL provides a single pass mapping solution avoiding pixel misalignment and minimising electron beam induced damage artefacts associated with multiple pass mapping. The instrument we have developed based upon a JEOL 8530F EPMA [3], includes a LN cold stage enabling spectroscopy to be performed at cryogenic temperatures. When acquiring a multi-spectral mapping dataset, the collection of SXES spectra is the rate limiting step, due to the time required to accumulate sufficient signal and then read the full CCD. In our case we employ a Princeton Instruments $2 \mathrm{~K} \times 2 \mathrm{~K}$ CCD camera. The fastest mapping currently undertaken is at $200 \mathrm{~ms}$ per pixel. EDS is collected using a Bruker windowless SDD, sensitive down to $50 \mathrm{eV}$.

To illustrate the mapping behaviour of this instrument we have collected a hyperspectral map on a sulphide mineral sample containing sphalerite $(\mathrm{ZnS})$ and pyrite $\left(\mathrm{FeS}_{2}\right)$. The map was collected at $7 \mathrm{kV}$, 150nA, 800ms dwell. The SXES had a 200N grating which provided an energy range of $64-350 \mathrm{eV}$, and the CL spectrometer had a grating sensitive from 200-950nm. In Figure 1, pyrite and sphalerite spectra have been extracted from the map and show the $\mathrm{C} \mathrm{K} 1^{\text {st }}-4^{\text {th }}$ order and S Ll $1^{\text {st }}$ and $2^{\text {nd }}$ order reflections. The S Ll emission in pyrite is comprised of up to four peaks spread over a $10 \mathrm{eV}$ energy range while in sphalerite it is spread over a smaller energy range and comprised of two main peaks. The pyrite in this sample was only weakly CL active, however the sphalerite yielded a CL spectra with a main peak at $2.2 \mathrm{eV}$, associated with $\mathrm{Mn}^{2+}$ and a minor peak at $1.35 \mathrm{eV}$ reflecting the bandgap, Figure 2. Interpretation of SXES and CL spectra, which may involve understanding a range of the material's properties, can be problematic, however for both techniques a similar set of approaches can be employed. In the first instance, when faced with an unknown material, the simplest approach would be to look at reference 
spectra collected from known standards. The building of libraries of reference spectra are already being created by groups such as Tohoku University and JEOL [4] for SXES and CSIRO for CL [5]. To progress further in the interpretation of spectra, careful peak fitting will need to be undertaken, and this will require a deeper understanding of the physics behind the peak shapes and the spectrometer response. Quantum mechanical based electronic structure calculations need to go beyond standard ground state density functional theory (DFT) calculations, and model excited states, using techniques such as time dependent DFT. [3]

\section{References:}

[1] M. Terauchi et al., 2012 Journal of Electron Microscopy 61(1), 1-8

[2] Burgess et al., 2014 Microscopy \& Microanalysis 20(Suppl. 3), 898-899

[3] We acknowledge support from ARC - LE130100087

[4] M. Terauchi et al., Handbook of Soft X-ray Emission Spectra, JEOL Ltd.

[5] C.M. MacRae and N.C. Wilson, 2008 Microscopy \& Microanalysis 14, 184-204

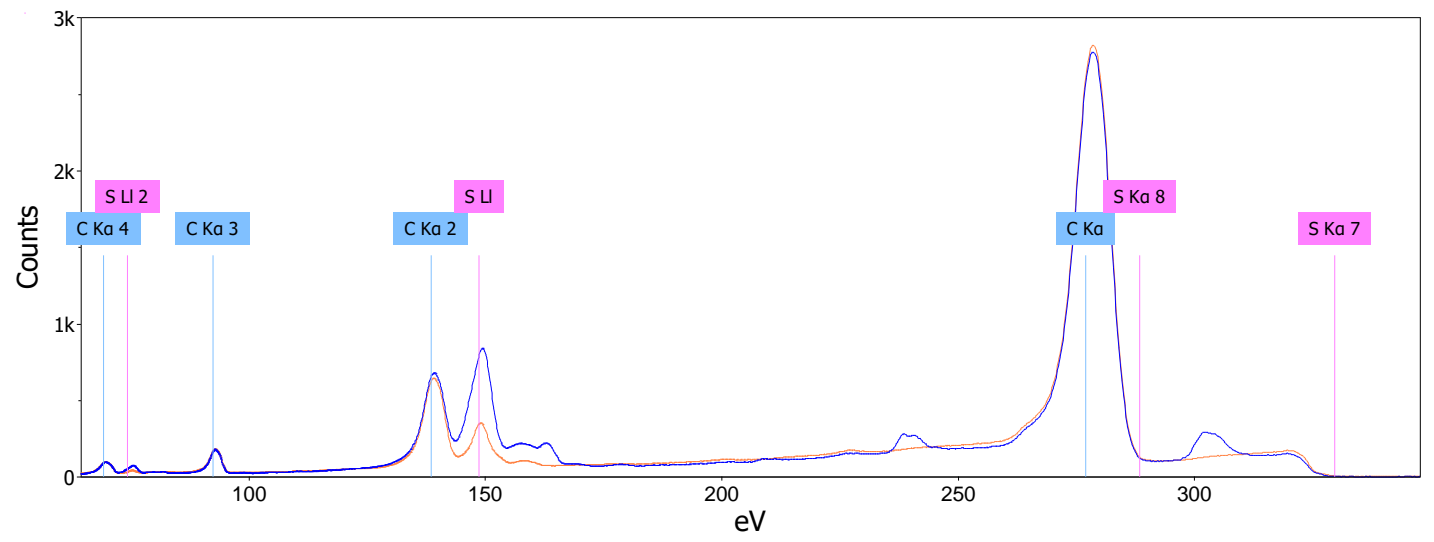

Figure 1. Soft X-ray spectra from pyrite (blue) and sphalerite (red), the $\mathrm{S} \mathrm{Ll}$ in pyrite is comprised of up to four peaks spread over a $10 \mathrm{eV}$ energy range.

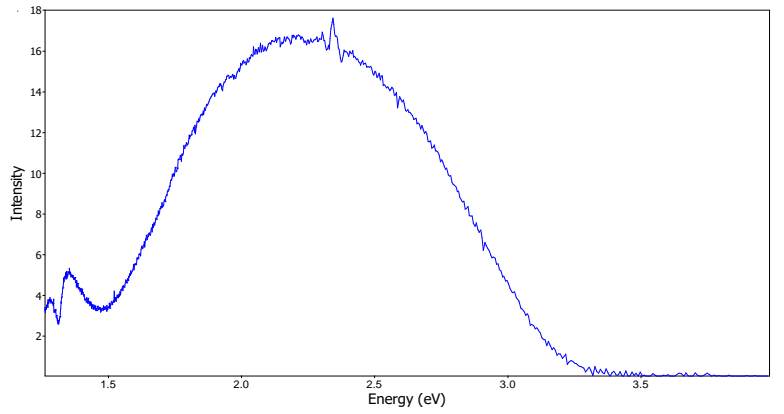

Figure 2. Cathodoluminescence spectra from sphalerite showing a main peak at $2.2 \mathrm{eV}$ most likely associated with $\mathrm{Mn}^{2+}$ and a peak at $1.35 \mathrm{eV}$ associated with the bandgap.

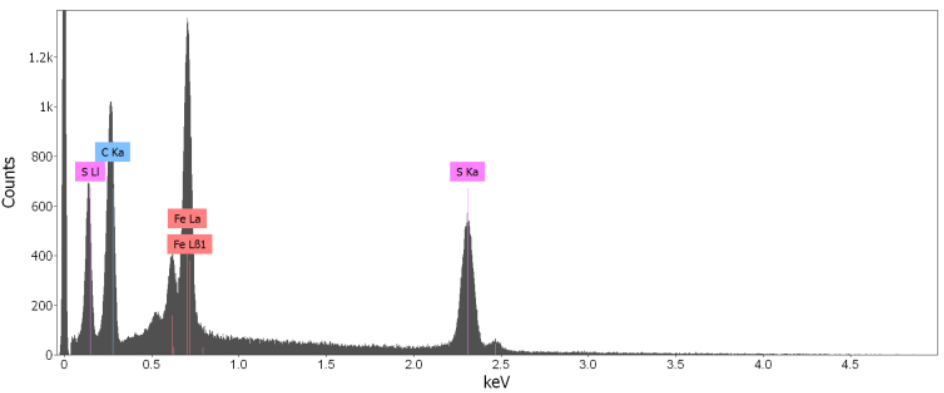

Figure 3. Pyrite spectrum showing the S Ll well resolved from the $\mathrm{C} \mathrm{K}$ peak. The ED spectrum enables fast identification of majors and minors in each phase analysed. 